\title{
Wave of translation
}

One day in 1834, Scottish naval engineer John Scott Russell was riding alongside a canal near Edinburgh, when two horses drawing a boat on the canal suddenly stopped. As the boat came to rest, it pushed up a broad swell of water, which set off down the canal, with a fascinated Russell giving chase. "I followed it on horseback," Russell reported, "and overtook it still rolling on at a rate of some eight or nine miles an hour, preserving its original figure some thirty feet long... after a chase of one or two miles I lost it in the windings of the channel. Such was my first-chance interview with that singular and beautiful phenomenon which I have called the Wave of Translation."

Russell began experimenting with such waves, and soon guessed a relationship between their speed $v$ and height $h$, depending on the depth of the channel $d: v=\sqrt{ } g(h$ $+d)$. Thirty-two years later, Lord Rayleigh derived this expression from basic hydrodynamics, confirming that Russell had discovered a fundamentally new and essentially nonlinear kind of wave-like excitation. Since then, our knowledge of solitons and other nonlinear waves has grown enormously, especially through the mathematics of the inverse scattering transform'. Yet we might still be closer to the beginning of a long exploration than to its end, with many surprises yet to come.

Take the curious 'intrinsically localized modes' (ILMs), a subject of growing interest. Numerous experiments and simulations at the macroscopic level demonstrate that a periodic array of elements with generic nonlinear restoring forces can support localized excitations, which are naturally stable and extend over a handful of lattice sites. Recent experiments with arrays of micromechanical cantilevers, for example, illustrate how an initially homogeneous excitation can grow unstable and decay into a small number of localized excitations, which persist and carry most of the vibrational energy (M. Sato et al. Rev. Mod. Phys. 78, 137-157; 2006). These localized excitations act more or less like particles, and interact strongly on close encounters, unlike traditional solitons, which pass through one another, suffering only a phase shift.

Similar ILMs have been observed in nonlinear photonic crystals and in arrays of Josephson

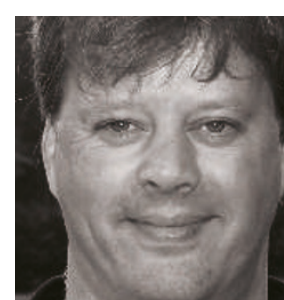

WE'VE LARGELY

FAILED TO

NOTICE

INHERENTLY

NONLINEAR

PHENOMENA. junctions. More intriguingly, numerous studies have found indirect traces of ILMs in realworld condensed-matter systems such as charge-transfer salts, quasione-dimensional ferromagnets and proteins. When we think of excitations in a crystalline solid lattice, we still think in habitually linear terms; nonlinearity tends to masquerade as linear excitations plus interactions. Consequently, it seems, we've largely failed to notice inherently nonlinear phenomena.

These localized objects bring to mind the beautiful 'oscillons' discovered several years ago as stable, particle-like excitations that can form on the surface of a vibrating bed of metallic beads. Might such structures play an important role in crystalline materials even in equilibrium? Based on current understanding, Sato et al. suggest conditions might be right in some special cases - in materials exhibiting paraelectric to ferroelectric phase transitions, for example. But we might ultimately find that ILM's, like Russell's 'wave of translation', have been with us for a long time - we just haven't got used to seeing them.

Mark Buchanan

\section{Proliferating madness}

No sane person would argue that the proliferation of nuclear weapons across more national borders would make the world a safer place, or that diminishing the psychological barrier against a renewal of nuclear testing, or the first use of nuclear weapons, is a good thing. Yet policies in Washington seem to be encouraging all of the above.

While railing against the possible development of nuclear capabilities in Iran, and refusing to negotiate with North Korea, Washington officials apparently kept secret their knowledge of a Pakistani initiative to build a new plutonium reactor that will be capable of producing up to 50 nuclear weapons a year. At the same time Washington has been pushing hard for a non-military nuclear cooperation pact with India that would help promote India's bomb-making capacity. Besides the incendiary possibilities of further arming two military rivals, it is important to remember that neither country has signed the Nuclear Nonproliferation Treaty. What message does that send to countries, such as Iran, that have signed the Treaty?

If this weren't bad enough, the US Congress is likely to nearly double funding for the Reliable Replacement Warhead programme, to develop and deploy new warheads to replace all existing weapons in the US nuclear arsenal. It is claimed that these warheads will not need testing, but most physicists familiar with

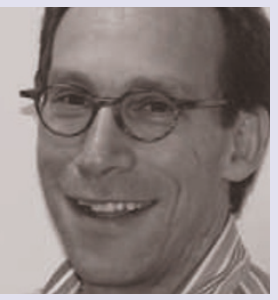

IF THE UNITED

STATES IS

RE-EXAMINING

ITS NUCLEAR

WEAPONS

ARSENAL,

SHOULDN'T

IT FIRST

RECONSIDER

WHY IT

HAS SUCH A

STOCKPILE? the programme recognize that there will be incredible pressure to do so, before deployment. This would put pressure on China, Russia, India and Pakistan to test their weapons and make it more difficult to convince Iran and North Korea to abandon their nascent programmes.

If the US is re-examining its nuclear weapons arsenal, shouldn't it first reconsider why it has a stockpile, in this post-coldwar world, of nearly 10,000 such weapons? There is no rational reason for preserving this, at incredible cost, in today's world. But then, rationality doesn't seem part of nuclear policy of late. Next month: first use, and what physicists should do about it.

Lawrence M. Krauss 